\title{
Damping of Nodal Fermions Caused by a Dissipative Mode
}

\author{
D. Schmeltzer \\ Physics Department \\ City College of the City University of New York \\ New York, New York 10031
}

(Dated: November 4, 2018)

\begin{abstract}
Using a $d_{x^{2}-y^{2}}$ superconductor in $2+1$ dimensions we show that the Nambu Goldstone fluctuations are replaced by dissipative excitations. We find that the nodal quasi-particles damping is caused by the strong dissipative excitations near the nodal points. As a result we find that the scattering rates are linear in frequency and not cubic as predicted in the literature for the "d" wave superconductors. Our results explain the recent angle resolved photoemission spectroscopy and optical conductivity in the BSCCO high $T_{c}$ compounds.
\end{abstract}

PACS numbers: 
The recent angle-resolved photoemission spectroscopy (ARPES) [1] and optical conductivity [2] studies of the superconducting high $T_{c}$ compound BSCCO show scattering rates which are linear in temperature $[1,2,3]$. Moving away from the nodal direction the scattering rates appear to level of and become temperature independent. These scattering rates are in strong contrast to the $\omega^{3}$ scattering suggested for the d-wave like pairing [4].

Our results are expected to hold for any $d_{x^{2}-y^{2}}$ superconductor in $2+1$ dimension. The fact that our predictions are only observed in BSCCO suggest that the YBCO superconductors have additional d-wave order parameters " $i d_{x y}$ ", "s" or have a large hopping in the "c" direction. In both situations the dissipative behavior caused by massless Dirac fermions in $2+1$ is absent!

Quasi-particles properties of nodal fermions in $d_{x^{2}-y^{2}}$ superconductors and $2+1$ dimensions are investigated. We find that the Nambu-Goldstone fluctuations or the massive plasma mode caused by the Coulomb interaction is replaced by a dissipative collective mode - a gauge invariant field. This result follows from the fact that the Nodal-Dirac [5-9] fermions which couples to a gauge field in $2+1$ dimension induces critical Q.E.D. and not regular photons [5-10]. We find that this dissipative mode causes the quasi-particle self energy in the vicinity of the nodal points to have scattering rate which are linear in frequency [1-3]. Moving away from the nodal point one finds that the scattering rate is reduced. When the d-wave order parameter has additional components, " $i d_{x y}$ ", "s", $a^{\prime}$ or single electrons hopping occurs in the "c" direction the scattering rate of the quasi-particles is suppressed.

In order to understand the origin of the dissipative mode we follow S. Weinberg [10] and replace the fermion operator $C_{\sigma}(x)$ by a neutral fermion field $\tilde{C}_{\sigma}(x)=\exp (-i \alpha(x) / 2) C_{\sigma}(x)$ where " $\alpha$ " is the Nambu-Goldstone phase. In the presence of an electromagnetic field $a_{\mu}^{e x t}$ one finds [10] that the electromagnetic response is represented in terms of the gauge invariant fields $a_{\mu}=\left(\partial_{\mu} \alpha-2 a_{\mu}^{e x t}\right)$. For any superconductor the electromagnetic response contains two parts, the diamagnetic contribution given by the vector potential square, $\frac{1}{2} \rho\left(\vec{\partial} \alpha-2 \vec{a}^{e x t}\right)^{2}(\rho$ is the electron density) and the paramagnetic response which is obtained by second order perturbation theory.

For an "s" wave superconductor the density-density response function is proportional to the superconducting density [12]. The current-current response function represents the parametric polarization, $\Gamma_{2}^{(i, j)}$ which at $T=0$ and $q \rightarrow 0$ vanishes, $\Gamma_{2}^{(i, j)}=0$ ! Therefore the diamagnetic term $\frac{1}{2} \rho\left(\vec{\partial} \alpha-2 \vec{a}^{e x t}\right)^{2}$ is not normalized. As a result the Lagrangian takes the 
form; $L^{s c} \sim \frac{1}{2} \rho\left[\frac{1}{v^{2}}\left(\partial_{t} \alpha-2 a_{0}^{e x t}\right)^{2}-\left(\vec{\partial} \alpha-2 \vec{a}^{e x t}\right)^{2}\right]$. The Lagrangian $L^{s c}$ gives rise to the low energy gapless mode - named Goldstone mode. The presence of the Coulomb interaction makes the Goldstone mode massive. This can be seen following the method presented in ref 12. The Coulomb interaction is replaced by a Hubbard-Stratonovich field $\tilde{a}_{0}$, which play the role of the temporal gauge field. $L^{s c+c} \sim \frac{1}{2} \rho\left[\frac{1}{v^{2}}\left(\partial_{t} \alpha-2 \tilde{a}_{0}\right)^{2}-(\vec{\partial} \alpha)^{2}\right]+\frac{1}{e^{2} 8 \pi}|\vec{q}|^{2} \tilde{a}_{0} \tilde{a}_{0}$ "e" is the electric charge and $|\vec{q}|$ is the momentum. Integration of the $\tilde{a}_{0}$ field changes the Goldstone mode into a massive mode!

The $d_{x^{2}-y^{2}}$ case in $2+1$ is different! In this case the paramagnetic response is not zero and gives rise to a dissipative mode which does'nt become massive in the presence of the Coulomb interaction. The difference is caused by the polarization diagram (see ref. 10, 12). Instead of $\Gamma_{2}^{(0,0)} \sim \rho_{s}$ and $\Gamma_{2}^{(i, j)}=0$ we have a branch-cut, $\Gamma_{2}^{(\mu, \nu)}(q)=\frac{1}{8 \sqrt{-q^{2}}}\left(-q^{2} g^{\mu \nu}+q^{\mu} q^{\nu}\right)$. As a result we have; $L^{d-\text { wave }} \sim \frac{1}{2}\left(\partial_{\mu} \alpha-2 a_{\mu}^{e x t}\right)_{\vec{q}, \omega} \Gamma_{2}^{(\mu, \nu)}(\vec{q}, \omega)\left(\partial_{\nu} \alpha-2 a_{\nu}^{e x t}\right)_{-\vec{q},-\omega}-\frac{1}{2} \rho\left(\vec{\partial} \alpha-2 \vec{a}^{e x t}\right)^{2}$. The most important difference in $L^{d-\text { wave }}$ is the fact that the term $\left(\partial_{t} \alpha-2 a_{0}^{e x t}\right)^{2}$ is absent, instead we have the branch-cut terms with the dissipative behavior! In order to consider the Coulomb interaction we use again the Hubbard Stratonovich field, $\tilde{a}_{\mu}=\delta_{\mu .0} \tilde{a}_{0}$.

$$
L^{d-\text { wave }} \sim \frac{1}{2}\left(\partial_{\mu} \alpha-2 \tilde{a}_{\mu}\right)_{\vec{q}, \omega} \Gamma_{2}^{(\mu, \nu)}(\vec{q}, \omega)\left(\partial_{\nu} \alpha-2 \tilde{a}_{\nu}\right)_{-\vec{q},-\omega}-\frac{1}{2} \rho(\vec{\partial} \alpha)^{2}+\frac{1}{e^{2} 8 \pi}|\vec{q}|^{2} \tilde{a}_{0} \tilde{a}_{0}
$$

Now the situation is different, instead $\Gamma_{2}^{(0,0)} \sim \rho$ and $\Gamma_{2}^{(i, i)} \sim 0$ we have; $\Gamma_{2}^{(0,0)} \sim \frac{2 \omega^{2}-|\vec{q}|^{2}}{8 \sqrt{-\omega^{2}+|\vec{q}|^{2}}}$ and $\Gamma_{2}^{(i, i)} \sim \frac{|\vec{q}|^{2}}{8 \sqrt{-\omega^{2}+|\vec{q}|^{2}}}$. As a result, dimensional analysis shows that the term $\frac{1}{2 e^{2}}|\vec{q}|^{2} \tilde{a}_{0} \tilde{a}_{0}$ is negligible with respect the term $\Gamma_{2}^{(0,0)}(q, \omega) \tilde{a}_{0} \tilde{a}_{0}$. For this reason we will ignore for the remaining part the effect of the Coulomb interaction.

Next we present our derivation. Our starting point is the nearest neighbor pairing action for a two-dimensional square lattice. We start with the superconductor action $\tilde{S}$ and the partition function $Z$.

$$
Z=\int D \Phi D \Phi^{*} D \tilde{C}_{\uparrow}^{\dagger} D \tilde{C}_{\uparrow} D \tilde{C}_{\downarrow}^{\dagger} D \tilde{C}_{\downarrow} \exp (i \tilde{S})
$$




$$
\begin{aligned}
\tilde{S} & =\int d t \sum_{r}\left\{\sum _ { \sigma = \uparrow , \downarrow } \left[\tilde{C}_{\sigma}^{\dagger}(r, t)\left(i \partial_{t}-a_{0}^{e x t}-E_{F}\right) \tilde{C}_{\sigma}(r, t)\right.\right. \\
& \left.+t \sum_{\mu=x, y}\left(\tilde{C}_{\sigma}^{\dagger}\left(r+d_{\mu}, t\right) \exp ^{i \int_{r+d_{\mu}}^{r} \vec{a}^{e x t} d \vec{r}} \tilde{C}_{\sigma}(r, t)+h . c .\right)\right] \\
& -\sum_{\mu=x, y}\left[\Phi\left(r, r+d_{\mu} ; t\right)\left(\tilde{C}_{\uparrow}^{\dagger}(r, t) \tilde{C}_{\downarrow}^{\dagger}\left(r+d_{\mu}, t\right)-\tilde{C}_{\downarrow}^{\dagger}(r, t) \tilde{C}_{\uparrow}^{\dagger}\left(r+d_{\mu}, t\right)\right)+h . c .\right] \\
& \left.+\frac{\left|\Phi\left(r, r+d_{\mu} ; t\right)\right|^{2}}{2 \lambda}\right\}
\end{aligned}
$$

The action $\tilde{S}$ is characterized by the nearest neighbor pairing fields $\Phi$ and $\Phi^{\star}$ with the coupling constant $\lambda$ and the external field $a_{\mu}^{e x t}$. We will perform a set of transformations which leave the partition function invariant. We start by replacing the Fermion field $\tilde{C}_{\sigma}(r)$ in terms of chiral fields $\tilde{R}_{r, \sigma}$ and $\tilde{L}_{r, \sigma}$ (the right and left chiral fermions). $\tau=1$ corresponds to the nodal liquid with $K_{F}=K_{\tau=1}=\left(\frac{\pi}{2 a}, \frac{\pi}{2 a}\right)$ and $\tau=2$ for $K_{F}=K_{\tau=2}=\left(\frac{\pi}{2 a},-\frac{\pi}{2 a}\right)$ (see Ref. $[5,6]$ ). $K_{\tau=1}$ corresponds to the Fermi surface with the normal in the direction $\hat{e}_{1}=(\hat{x}+\hat{y}) / \sqrt{2}$ and $\tau=2$ is rotated by $90^{\circ}$ into the direction $\hat{e}_{2}=(\hat{x}-\hat{y}) / \sqrt{2}$. We replace the Fermion field by $\tilde{C}_{\sigma}(r, t)=\sum_{r=1,2}\left[\exp \left(i \vec{K}_{\tau} \cdot \vec{r}\right) \tilde{R}_{\tau, \sigma}(r, t)+\exp \left(-i \vec{K}_{\tau} \cdot \vec{r}\right) \tilde{L}_{\tau, \sigma}(r, t)\right]$. Following Ref. [11] we have parametrized the superconductor order parameter $\Phi$ in terms of the amplitude $\rho(r)$ and the "Nambu Goldstone" phase $\alpha(r)$. This leads to a change of the measure from $D \Phi D \Phi^{\star}$ to $D \alpha D \rho$. We take the saddle point of the action $\tilde{S}$ and find that the minimum occurs for $\rho\left(r, r+d_{x}\right)=-\rho\left(r, r+d_{y}\right)$ which corresponds to the $d_{x^{2}-y^{2}}$ symmetry. We obtain for the saddle point, $\Delta=2 \sqrt{2}|\rho|=\left(\frac{2}{\pi}\right)^{2} \frac{\hat{\lambda}}{3}$. In agreement with Ref. [5] we introduce the spinors $\tilde{\psi}_{\tau}^{\dagger}=\left(\tilde{R}_{\tau, \uparrow}^{\dagger}, \tilde{L}_{\tau, \downarrow}^{\dagger}\right)$ and $\tilde{\chi}_{\tau}^{\dagger}=\left(\tilde{R}_{\tau, \uparrow}^{\dagger},-\tilde{L}_{\tau, \downarrow}^{\dagger}\right)$ with $\tau=1,2$. In terms of these spinors we obtain two Dirac Fermion representations with the Cartesian axes rotated.

Next we perform a gauge transformation [6] which replaces the spinor fields $\tilde{\psi}_{\tau}$ and $\tilde{\chi}_{\tau}$ by the neutral nodal fermions $\psi_{\tau}$ and $\chi_{\tau}$.

$$
\begin{gathered}
\psi_{\tau}(r, t)=\exp \left(-i \frac{\sigma_{3}}{2} \alpha(r, t)\right) \tilde{\psi}_{\tau}(r, t) \\
\chi_{\tau}(r, t)=\exp \left(-i \frac{\sigma_{3}}{2} \alpha(r, t)\right) \tilde{\chi}_{\tau}(r, t) \\
\Phi\left(r, r+d_{\mu} ; t\right)=\exp \left(\frac{i}{2}(\alpha r, t)\right) \rho\left(r, r+d_{\mu} ; t\right) \exp \left[\frac{i}{2} \alpha\left(r+d_{\mu}, t\right)\right]
\end{gathered}
$$


From eq. 2a we learn that the spinor field is given as a product of the neutral spinor and the boson field $\exp \left(e^{-i \sigma_{3}} \frac{\alpha}{2}(r, t)\right)$. In terms of the fermions fields we have $\tilde{R}_{\tau, \sigma}=e^{i \frac{\alpha}{2}} R_{\tau, \sigma}$ and $\tilde{L}_{\tau, \sigma}=e^{i \frac{\alpha}{2}} L_{\tau, \sigma}$ where $e^{i \alpha / 2}$ carries the charge of a half Cooper pair.

As a result of the gauge transformation given by Eq. (2) the action $\tilde{S}$ is replaced by $\hat{S}$. We express the action $\hat{S}$ in terms of the spinors $\psi_{\tau}, \bar{\psi}_{\tau}=\psi_{\tau}^{\dagger} \gamma^{0}, \chi_{\tau}$, and $\bar{\chi}_{\tau}=\chi_{\tau}^{\dagger} \gamma^{0}$, where $\gamma^{0}$ is the Dirac gamma matrix. The Dirac action is obtained with the help of the derivative expansion. We obtain in the continuum limit two parts: The first part is linear in the gauge fields $\left(\partial_{\mu} \alpha-2 a_{\mu}^{e x t}\right)$ and is given by two Dirac actions $\hat{S}_{0}^{(\tau)}, \tau=1,2$ (the two directions see refs 5,6$)$. The second part is proportional to $\left(\partial_{\mu} \alpha-2 a_{\mu}^{e x t}\right)^{2}$ and represents the diamagnetic action, $S_{d i a}^{(\tau)}$.

The Dirac action is characterized by two velocities, $v_{1,1}=v_{2,2}=1$ and $v_{1,2}=v_{2,1}=\Delta \equiv$ $\frac{\tilde{\Delta}}{2 \sqrt{2 t}}$ (The first index corresponds to the nodal liquid $\tau=1,2$ and the second one to the direction $\mu=1,2)$. We take the expectation value of $\left\langle S_{\text {dia }}^{(\tau)}\right\rangle$ with respect to the free Fermion and generate a diamagnetic term [see Eq. (4)]. The diamagnetic mass in Eq. (4) is given by $r_{0}(\Delta) \equiv \Lambda \hat{r}_{0}, \hat{r}_{0}=\frac{1}{6 \pi} \int_{0}^{2 \pi} \frac{d \theta}{2 \pi} \frac{\cos ^{2} \theta}{\sqrt{1+\left(1-\Delta^{2}\right) \sin ^{2} \theta}}$, where " $\Lambda$ " is the ultraviolet cutoff. The explicit calculation of the diamagnetic term is given in ref. 6. See in particular eqs. 14, 19, 27 and 28 in ref. 6 .

For the remaining part we restrict the calculation to $\Delta \neq 0$ and as a result we obtain two Dirac equations coupled to gauge fields in $2+1$ dimensions: $\hat{S}=\hat{S}_{0}^{\tau}+\hat{S}_{\text {dia }}^{\tau}, \tau=1,2$,

$$
\begin{gathered}
\hat{S}_{0}^{\tau}=\int d^{d} x\left[\bar{\psi}_{\tau}\left(i \tilde{\tilde{\partial}}-m+A_{\tau}\right) \psi_{\tau}+\bar{\chi}_{\tau}\left(i \tilde{\tilde{\partial}}-m+A_{\tau}\right) \chi_{\tau}\right], \quad m \rightarrow 0 \\
\hat{S}_{d i a}^{\tau}=\int d^{d} x\left\{-\frac{r_{0}(\Delta)}{2}\left(A_{\tau, 0}\right)^{2}\right\}, \quad d^{d} x \equiv d^{2} x d t
\end{gathered}
$$

where $A_{\tau, \mu}=\gamma^{\mu} A_{\tau, \mu}, \tilde{\partial}=v_{\tau, \mu} \gamma^{\mu} \partial_{\mu}, v_{\tau, \mu}=\left(1, v_{\tau, 1}, v_{\tau, 2}\right), v_{1,1}=v_{2,2}=1, v_{1,2}=v_{2,1}=\Delta$. The form of Eqs. (3) and (4) has been obtained after performing a derivative expansion in the action in eq. $1 . A_{\tau, 0}^{2}$ in eq. 4 is the spatial component of the gauge invariant field $A_{\mu}$.

Eq. (3) represents the neutral Dirac equation coupled to a gauge field $A_{\tau}$ which is a gauge invariant quantity. Therefore, the neutral particles $\psi_{\tau}$ and $\chi_{\tau}$ couples to the gauge invariant field, $A_{0}$. The gauge invariant form follows from the combination of the Nambu Goldstone phase and the vector potential $\vec{a}_{\mu}^{e x t}$. 


$$
\begin{aligned}
& A_{\tau=1,0}=a_{1}=\left(a_{1}^{e x t}-\frac{1}{2} \partial_{1} \alpha\right) \\
& A_{\tau=2,0}=a_{2}=\left(a_{2}^{e x t}-\frac{1}{2} \partial_{2} \alpha\right) \\
& A_{\tau=1,1}=A_{\tau=2,2}=a_{0}=\left(a_{0}^{e x t}-\frac{1}{2} \partial_{t} \alpha\right) \\
& A_{\tau=1,2}=A_{\tau=1,2}=0
\end{aligned}
$$

It is important to mention that the gauge field in eq. 3 is similar to the gauge fields in $1+1$ dimensions [9] the reason being that the second component is zero $A_{\tau=1,2}=A_{\tau=2,1}=0$, (the index $\tau=1,2$ corresponds to the Fermi surface and the second index represents the cartesian direction). Combining the two gauge fields $A_{\tau, \mu}, \tau=1,2$, we obtain a gauge field in $2+1$ dimensions, $A_{\tau=1,0} \equiv a_{1}, A_{\tau=2,0} \equiv a_{2}, A_{\tau=1,1}=A_{\tau=2,2} \equiv a_{0}$. The diamagnetic term in eq. 4 give rise to a Meisner effect, controlled by the penetration depth, $\left(r_{0}(\Delta)\right)^{-1}$ evaluated in eqs. 27,28 in ref. 6.

Next we will compute the single particle Green's function. We will consider first the neutral part.

$$
\begin{aligned}
& G_{\tau=1}(r, t)=\left\langle\left\langle T \psi_{\tau=1}(r, t) \psi_{\tau=1}^{\dagger}(0,0)\right\rangle\right\rangle \\
& =\frac{1}{N} \int D A_{\tau=1} D A_{\tau=2}\left(i \gamma^{0} \check{\partial}+\gamma^{0} A_{\tau=1}\right)_{r, t ; 0,0}^{-1} \quad e^{i \hat{S}_{d i a}^{(\tau=1)}} \\
& \cdot\left(\operatorname{det}\left(i \tilde{\widetilde{\delta}}+A_{\tau=1}\right) \operatorname{det}\left(i \tilde{\widetilde{\partial}}+A_{\tau=2}\right)\right)^{2} \quad e^{i \hat{S}_{d i a}^{(\tau=2)}}
\end{aligned}
$$

The presence of $\gamma^{0}$ in eq. 6 is due to the fact that we compute $\psi_{\tau}(2) \psi_{\tau}^{\dagger}(1)=\psi_{\tau}(2) \bar{\psi}_{\tau}(1) \gamma^{0}$ and not $\psi_{\tau}(2) \bar{\psi}_{\tau}(1)$. "N" represents the normalization constant. In order to find the single particle Green's function in eq. 6 we have to evaluate the determinant; $\operatorname{det}\left(i \tilde{\widetilde{\partial}}+A_{\tau}\right)=$ $\exp i \hat{S}_{e f f}\left(A_{\tau}\right)$.

$$
\hat{S}_{\text {eff }}\left(A_{\tau}\right)=\lim _{m \rightarrow 0} i \sum_{n=1}^{\infty} \frac{1}{n}\left\{\operatorname{Tr}\left[\frac{i}{i \tilde{\widetilde{\partial}}-m}\left(-i A_{\tau}\right)\right]^{n}\right\}=\hat{S}_{2}\left(A_{\tau}\right)+\hat{S}_{I}\left(A_{\tau}\right)
$$

where

$$
\hat{S}_{2}\left(A_{\tau}\right)=\frac{1}{2} \int^{\Lambda} d^{d} q A_{\tau, \mu}(q) \Gamma_{2}^{\mu \nu}(q) A_{\tau, \nu}(-q)
$$

with

$$
\Gamma_{2}^{\mu \nu}(q)=2 \lim _{m \rightarrow 0} \int^{\Lambda} d^{d} k \operatorname{Tr}\left[\frac{\gamma^{\mu}(k+m) \gamma^{\nu}(k+q+m)}{\left(k^{2}-m^{2}\right)\left[(k+q)^{2}-m^{2}\right]}\right]=\frac{1}{8 \sqrt{-q^{2}}}\left(-q^{2} g^{\mu \nu}+q^{\mu} q^{\nu}\right)
$$


$\hat{S}_{I}\left(A_{\tau}\right)$ represents the non-Gaussian part. In eq. (9) we used the notation, $g^{\mu \nu}=0$ for $\mu \neq \nu$ and $g^{00}=g^{i i}=1$. For the momentum $q$ we use $q^{\mu}=\left(q^{0}, \vec{q}\right), q^{0}=\omega$ and $q^{2}=q^{\mu} q_{\mu}=\left(q^{0}\right)^{2}-(\vec{q})^{2}$. In eq. (9) we observe that the "branch-cut" for $\omega /|\vec{q}|>1$ gives rise to an imaginary polarization [10]. This describes the threshold for destroying the electron pairs and creating normal electrons.

We combine $\Gamma_{2}^{\mu \nu}(q)$ given in eq. 7 with the diamagnetic part $\hat{S}_{\text {dia }}^{(\tau)}$ and find the low energy action in the gauge invariant form.

$$
\hat{S}_{2, e f f}\left(A_{\mu}\right)=\frac{1}{2} \int^{\Lambda} d^{d} q A_{\mu}(q) \hat{\Gamma}_{2}^{\mu \nu}(q) A_{\mu}(-q)
$$

where

$$
\begin{aligned}
& \hat{\Gamma}_{2}^{(0,0)}(q)=\Gamma_{2}^{(1,1)}(q)+\Gamma_{2}^{(2,2)}(q), \quad \bar{\Gamma}_{2}(q) \equiv \hat{\Gamma}_{2}^{(1,1)}(q)=\hat{\Gamma}_{2}^{(2,2)}(q)=\Gamma_{2}^{(0,0)}(q)-r_{0}(\Delta) \\
& \hat{\Gamma}_{2}^{(0,2)}(q)=\Gamma_{1}^{(2,0)}(q), \quad \hat{\Gamma}_{2}^{(0,1)}(q)=\Gamma_{2}^{(1,0)}(q)
\end{aligned}
$$

In eqs. $10,11 \hat{\Gamma}_{2}^{(\mu, \nu)}(q)$ is the vertex for the fields $a_{\mu}$ expressed in terms of the original vertex $\Gamma_{2}^{(\mu, \nu)}(q)$ (For the fields $A_{\tau, \mu}$ see eqs 8,9).

Using eq. 10 we obtain the "photon" Green's function.

$$
\begin{aligned}
& D_{00}(\vec{q}, \omega)=\left\langle a_{0}(\vec{q}, \omega) a_{0}(-\vec{q},-\omega)\right\rangle \\
& =-i \Delta\left[\frac{\omega^{2}-\left(\Delta q_{2}\right)^{2}}{8\left(-\omega^{2}+q_{1}^{2}+\left(\Delta q_{2}\right)^{2}-i \epsilon\right)^{1 / 2}}+\frac{\omega^{2}-\left(\Delta q_{1}\right)^{2}}{8\left(-\omega^{2}+q_{2}^{2}+\left(\Delta q_{1}\right)^{2}-i \epsilon\right)^{1 / 2}}\right]^{-1} \\
& \underset{\Delta \rightarrow 1}{\longrightarrow} \frac{-i \Delta 8\left(-\omega^{2}+|\vec{q}|^{2}-i \epsilon\right)^{1 / 2}}{2 \omega^{2}-|\vec{q}|^{2}} \\
& D_{11}(\vec{q}, \omega)=\left\langle a_{1}(\vec{q}, \omega) a_{1}(-\vec{q},-\omega)\right\rangle \\
& =i q_{2}^{2} \Delta\left[\left(\frac{q_{2}}{|\vec{q}|}\right)^{2}\left(\frac{q_{1}^{2}+\left(\Delta q_{2}\right)^{2}}{8\left(-\omega^{2}+q_{1}^{2}+\left(\Delta q_{2}\right)^{2}-i \epsilon\right)^{1 / 2}}-r_{0}(\Delta)\right)\right. \\
& \left.+\left(\frac{q_{1}}{|\vec{q}|}\right)^{2}\left(\frac{q_{2}^{2}+\left(\Delta q_{1}\right)^{2}}{8\left(-\omega^{2}+q_{2}^{2}+\left(\Delta q_{1}\right)^{2}-i \epsilon\right)^{1 / 2}}-r_{0}(\Delta)\right)\right]^{-1} \\
& \underset{\Delta \rightarrow 1}{\longrightarrow} i 8 \Delta\left(\frac{q_{2}}{|\vec{q}|}\right)^{2} \frac{\left(-\omega^{2}+|\vec{q}|^{2}-i \epsilon\right)^{1 / 2}}{|\vec{q}|^{2}-8 r_{0}(\Delta)\left(-\omega^{2}+|\vec{q}|^{2}-i \epsilon\right)^{3 / 2}} \\
& D_{22}(\vec{q}, \omega)=\left\langle a_{2}(\vec{q}, \omega) a_{2}(-\vec{q},-\omega)\right\rangle \\
& \underset{\Delta \rightarrow 1}{\longrightarrow} i \Delta 8\left(\frac{q_{1}}{|\vec{q}|}\right)^{2} \frac{\left(-\omega^{2}+|\vec{q}|^{2}-i \epsilon\right)^{1 / 2}}{|\vec{q}|^{2}-8 r_{0}(\Delta)\left(-\omega^{2}+|\vec{q}|^{2}-i \epsilon\right)^{1 / 2}}
\end{aligned}
$$


In the absence of the gauge field $A_{\tau}$ the neutral fermion Green's function is given by $G_{\tau=1}^{0}(\vec{q}, \omega)=\frac{i\left(\omega I+\sigma_{3} \nu q_{1}-\sigma_{1} \Delta q_{2}\right.}{\omega^{2}-\left(\nu q_{1}\right)^{2}-\left(\Delta q_{2}\right)^{2}}$. Using the representation in eq. 6 and the dissipative photon Green's functions given in eqs 12-14, we compute the fermion self energy. to the one loop approximation we find that the self energy is given by $\Sigma_{\tau}(\vec{q}, \omega)$.

$$
\Sigma_{\tau=1}(\vec{q}, \omega)=\omega \int^{\Lambda} \frac{d^{2} k}{(2 \pi)^{2}} \int \frac{d \Omega}{2 \pi} \frac{1}{(\Omega-\omega)^{2}-(\vec{k}-\vec{q})^{2}}\left[D_{11}(\vec{k}, \Omega)+D_{00}(\vec{k}, \Omega)\right]
$$

The scattering rate in the vicinity of the nodal points, $|\vec{q}| \approx 0$ is given by the imaginary part of eq. 15 . The main contribution is due to the $D_{00}(\vec{k}, \Omega)$ photon. Performing a contour integral with the pole at $\Omega=\omega+|\vec{k}-\vec{q}| \sim \omega+|\vec{k}|$, we find in the limit of $\Delta \rightarrow 1$ the result;

$$
\operatorname{Im} \Sigma_{\tau=1}(\vec{q} \approx 0, \omega)=\frac{\omega^{2} \Delta}{\pi} \int_{1}^{1+\Lambda / \omega} d y \frac{(2 y-1)^{1 / 2}}{1+y^{2}}=\frac{4 \omega \Delta}{\pi} F\left(\frac{\omega}{\Lambda}\right) ; \quad F\left(\frac{\omega}{\Lambda}\right) \approx 1-\left(\frac{\omega}{\Lambda}\right)^{1 / 2}+\cdots
$$

Eq. 16 has been obtained in the limit $\Delta \rightarrow 1$. The reason for this being that according to eq. 12 the calculation becomes simple in this limit. Eq. 16 represents the " $\omega$ " dependence of the scattering rate at $T=0$. This result is much stronger than the $\omega^{3}$ result known in the literature [3].

At finite temperatures $T<\Lambda$ eq. 16 gives for the scattering rate, $\frac{1}{\tau} \sim \frac{T \Delta}{\pi} F\left(\frac{T}{\Lambda}\right)$. Moving away from the nodal point $|\vec{q}| \simeq 0$ gives a reduced scattering rate for $|\vec{q}|>\omega$, $\frac{1}{\tau} \sim \frac{\omega \Delta}{\pi}\left[\left(\frac{\omega}{|\vec{q}|}\right)^{2}-\left(\frac{\omega}{\Lambda}\right)^{2}\right]$. These results explain the recent ARPES [1] and conductivity [2] experiments.

Next we consider the Green's function for the quasi-particles,

$$
\tilde{G}_{\tau}(r, t)=\left\langle\left\langle T \tilde{\psi}_{\tau}(r, t) \tilde{\psi}_{\tau}^{\dagger}(0)\right\rangle\right\rangle \simeq\left\langle e^{-\frac{i \sigma_{3}}{2}(\alpha(r, t)-\alpha(0))}\right\rangle_{A} G_{\tau}(r, t)
$$

The phase $\alpha(r, t)$ is given in terms of the gauge field $a_{\mu}\left(\right.$ for $\left.a_{\mu}^{e x t}=0\right)$.

Integration of eqs 5 with the condition $a_{\mu}^{e x t}$ gives for the Nambu Goldstone phase the result: $\alpha(x, y, t)=2\left[\int_{0}^{t} a_{0}\left(0,0 ; t^{\prime}\right) d t^{\prime}+\int_{0}^{x} a_{1}\left(x^{\prime}, 0 ; t\right) d x^{\prime}+\int_{0}^{y} a_{2}\left(x, y^{\prime} ; t\right) d y^{\prime}\right]$. Using this representation we compute $C(r ; t) \equiv\left\langle\exp \left(-\frac{i \sigma_{3}}{2}(\alpha(\vec{r}, t)-\alpha(0 ; 0))\right\rangle_{a}\right.$. For simplicity we evaluate the equal time correlation $C\left(\vec{R}=R_{x}, R_{y} ; 0\right)$, 


$$
\begin{aligned}
C\left(\vec{R}=R_{x}, R_{y} ; 0\right) & \approx \exp \left[-\frac{1}{4}\left\langle\left(\alpha\left(x+R_{x}, y+R_{y} ; t\right)-\alpha(x, y ; t)^{2}\right)^{2}\right\rangle\right] \\
& =\exp \left[-\frac{1}{2} \int d w \int d^{2} q\left[D_{11}(\vec{q}, \omega) \frac{1-\cos q_{x} R_{x}}{q_{x}^{2}}-D_{22}(\vec{q}, \omega) \frac{1+\cos q_{y} R_{y}}{q_{y}^{2}}\right]\right. \\
& \overrightarrow{\Delta \rightarrow 1} \exp \left[-\frac{8}{\pi^{2}} \hat{r}_{0}(\Delta) \hat{R}^{2}\right] ; \quad \hat{R}=\sqrt{\left(\frac{R_{x}}{a}\right)^{2}+\left(\frac{R_{y}}{a}\right)^{2}}
\end{aligned}
$$

" $a$ " is the lattice constant and " $\hat{r}_{0}(\Delta)$ " is the dimensionless inverse penetration length. Due to the fact that the correlation $C(\vec{R})$ is short range allows us to replace the Green's function $\tilde{G}_{\tau}$ with the neutral one $G_{\tau}(r, t), G_{\tau}(r, t) \approx \tilde{G}_{\tau}(r, t)$. This replacement is justified for distances $\left.r \leq \frac{\pi}{\sqrt{2}}\left(\frac{1}{\hat{r}_{0}(\Delta)}\right)^{1 / 2}\right)$ smaller than the pair correlation function.

Next we want to comment on the effect of the nonlinear term $\hat{S}_{I}\left(A_{\tau}\right)$ (given in eq. 7). Dimensional analysis suggests that our problem in $2+1$ dimensions is equivalent to Q.E.D. at $3+1$ dimensions. This shift in dimensionality follows from eqs. 8,9. To the one loop approximation the nonlinear terms $\hat{S}_{I}\left(A_{\tau}\right)$ renormalizes $[13,14]$ the vertex $\Gamma_{2}^{\mu \nu}(q)$ (see eq. 3 ) to $\hat{\Gamma}_{2}^{\mu \nu}(q) \sim \frac{1}{8}\left[1+\frac{14}{3 \pi^{2}} \ln \left(\frac{\Lambda}{-q^{2}}\right)\right]^{1 / 2} \cdot \Gamma_{2}^{\mu \nu}(q)$. Therefore the use of the normalized vertex $\hat{\Gamma}_{2}^{\mu \nu}(q)$ will not change significantly the result in eq. 11.

In the last part we consider the effect of the Coulomb interaction and show that it can be ignored. Following ref. 12 we replace the Coulomb term by a Hubbard field $\tilde{a}_{0}$ with the action $\frac{1}{2 e^{2}} \frac{|\vec{q}|^{2}}{4 \pi} \tilde{a}_{0}(\vec{q}, \omega) \tilde{a}_{0}(-\vec{q},-\omega)$. $\tilde{a}_{0}$ plays the role of the scalar gauge field. We replace in eq. $5, \vec{a}^{\text {ext }}=0, a_{0}^{\text {ext }}=\tilde{a}_{0}$ and find for the Nambu Goldstone phase " $\alpha$ " couples to the scalar field $\tilde{a}_{0}$ the Lagrangian $L\left(\alpha, \tilde{a}_{0}\right)$

$$
\begin{aligned}
L\left(\alpha, \tilde{a}_{0}\right) & =\alpha(\vec{q}, \omega)\left[\omega^{2} \hat{\Gamma}_{2}^{(0,0)}(\vec{q}, \omega)+|\vec{q}|^{2} \bar{\Gamma}_{2}(\vec{q}, \omega)+\omega q_{1} \hat{\Gamma}_{2}^{(0,1)}(\vec{q}, \omega)+\omega q_{2} \hat{\Gamma}_{2}^{(0,2)}(\vec{q}, \omega)\right] \alpha(-\vec{q},-\omega) \\
& +\frac{1}{2} \tilde{a}_{0}(\vec{q}, \omega)\left[e^{2} \hat{\Gamma}_{2}^{(0,0)}(\vec{q}, \omega)+\frac{|\vec{q}|^{2}}{4 \pi}\right] \tilde{a}_{0}(-\vec{q},-\omega) \\
& +\frac{1}{2} \alpha(\vec{q}, \omega)\left[e(i \omega) \hat{\Gamma}_{2}^{(0,0)}(\vec{q}, \omega)-i q_{1} \hat{\Gamma}_{2}^{(0,1)}(\vec{q}, \omega)-i q_{2} \hat{\Gamma}_{2}^{(0,2)}(\vec{q}, \omega)\right] \tilde{a}_{0}(-\vec{q},-\omega)+\text { h.c. }(19)
\end{aligned}
$$

The explicit form of the parameters in eq. 19 are obtained from eq. 11. In the limit $\Delta \rightarrow 1$ we obtain: $\hat{\Gamma}_{2}^{(0,0)}(\vec{q}, \omega)=\left(2 \omega^{2}-|\vec{q}|^{2}\right) \gamma(\vec{q}, \omega), \bar{\Gamma}_{2}(\vec{q}, \omega)=|\vec{q}|^{2} \gamma(\vec{q}, \omega)-r_{0}(\Delta) ; \hat{\Gamma}_{2}^{(0, i)}(\vec{q}, \omega)=$ $\omega q_{i} \gamma(\vec{q}, \omega), i=1,2 ; \gamma(\vec{q}, \omega)=\frac{1}{8}\left(-\omega^{2}+|\vec{q}|^{2}\right)^{-1 / 2}$. From eq. 19 we observe that contrary to the "s" wave case (ref 12) the vertex $e^{2} \hat{\Gamma}^{(0,0)}(\vec{q}, \omega)+\frac{|\vec{q}|^{2}}{4 \pi} \underset{q \rightarrow 0}{\longrightarrow}-\frac{i e^{2} \omega}{4}$ not a constant (see ref. 12). Therefore the excitations will remain massless in the presence of Coulomb interactions. 
In order to obtain the Nambu-Goldstone excitations we integrate the field $\tilde{a}_{0}$ and find:

$$
\begin{aligned}
& L(\alpha)=\frac{1}{2} \alpha(q, \omega)\left\{| \vec { q } | ^ { 2 } \left[\omega^{2}\left(\frac{\hat{\Gamma}^{(0,0)}(\vec{q}, \omega)\left(e^{2} 4 \pi\right)^{-1}-\gamma^{2}(\vec{q}, \omega)}{\hat{\Gamma}^{(0,0)}(\vec{q}, \omega)+\frac{|\vec{q}|^{2}}{e^{2} 4 \pi}}+\gamma(\vec{q}, \omega)\right)\right.\right. \\
& \left.\left.-r_{0}(\Delta)+|\vec{q}|^{2} \gamma(\vec{q}, \omega)\right]\right\} \alpha(-\vec{q},-\omega)
\end{aligned}
$$

In the limit $|\vec{q}| \rightarrow 0$ we look for massless solutions $\omega=z|\vec{q}|$. We substitute $\omega=z|\vec{q}|$ into the last equation and find a polynomial $P(z)$ for " $z$ ";

$P(z)=z^{2}\left(2 z^{2}-1\right)\left(1+\left(e^{2} 4 \pi\right)^{-1}\left(1-z^{2}\right)^{1 / 2}\right)-z^{2}-r_{0}(\Delta)\left(1-z^{2}\right)^{1 / 2}\left(2 z^{2}-1\right)=0$. Massless

solution exists for $z=z\left(r_{0}\right) \approx \sqrt{\frac{r_{0}(\Delta)}{3\left(e^{2} 4 \pi\right)^{-1}-1}}$. We find finite solutions for $z \approx z\left(r_{0}\right)$ (real and imaginary.), therefore the excitations are massless in the presence of Coulomb interactions.

To conclude, a new explanation for the scattering rate is presented. Instead of the phenomenological explanation given in refs 15,8 , we show that the neutral quasi-particles are scattered by the dissipative collective excitations giving rise to a linear temperature damping at the nodal points, in agreement with the ARPES [1] and conductivity $[2,3]$ experiments. The absence of the linear scattering rate in the YBCO superconductor might be due to the absence of the dissipative mode caused by the hopping in the ' $\mathrm{c}$ ' direction.

[1] T. Valla et al., Science 285, 2110 (1999); Phys. Rev. Lett. 85, 828(2000); A. Kaminsky et al., ibid. 84, 1788 (2000).

[2] J. Carson et al., Phys. Rev. Lett. 85, 2569 (2000).

[3] J. Carson, J. Orenstein, Seongshik Oh, J. O’Donnell,and J.N. Eeckstein, Phys. Rev. Lett. 85, 2569 (2000).

[4] S.M. Quinlan, D.J. Scalapino,and N. Bulut, Phys. Rev. B 49, 1470 (1994).

[5] L. Balents, M.P.A. Fisher,and C. Nayak, Int. J. Mod. Phys. B 12, 1038 (1988).

[6] D. Schmeltzer, J. Phys. Condens. Matter 13, 1699 (2001).

[7] M. Franz,and Z. Tešanović, Phys. Rev. Lett. 87, 257003 (2001).

[8] D.V. Khveshchenko ,and J. Paaske, Phys. Rev. Lett. 86, 4672 (2001).

[9] Steven H. Simon,and Patrick A. Lee, Phys. Rev. Lett. 78, 1548 (1997).

[10] R. Jackiw,and S. Templeton, Phys. Rev. D 23, 2291 (1988).

[11] S. Weinberg, "The Quantum Theory of Fields", vol. 2, pages 415-423, Cambridge University Press (1996). 
[12] N. Nagaosa, "Quantum Field Theory in Condensed Matter Physics", pages 129-133, Springer Verlag (1999).

[13] C. Itzykson,and J. Zuber, "Quantum Field Theory", pages 415-423.

[14] D. Schmeltzer, to appear in Physics Letter A., (2001).

[15] M. Vojta, Y. Zhang,and S. Sachdev, Phys. Rev. Lett. 85, 4940 (2000). 